\title{
EDITORIAL
}

\section{Ultrasound stethoscopy: a renaissance of the physical examination?}

\section{J R T C Roelandt}

The availability and versatility of echo/Doppler has made it the most widely used test to diagnose and quantify heart disease in many different health care environments. What impact will hand held ultrasound imaging devices have on our future diagnostic capabilities?

Correspondence to: Professor Jos RTC Roelandt Department of Cardiology, Thoraxcentre, Erasmus MC Rotterdam, 3015 GD, The Netherlands;

j.r.t.c.roelandt@erasmusmc.n
T his year marks the 50th anniversary of echocardiography. The evolution of this noninvasive imaging modality has been impressive and has paralleled the rapid developments in microprocessor technology. Together with Doppler assessment of intracardiac haemodynamics, a comprehensive and diagnostic evaluation of most patients suspected of having heart disease is now possible. The method introduced new pathophysiologic concepts and has made unique contributions to the management of cardiac patients. Because of its availability and versatility in application, echo/Doppler has become the most widely used test to diagnose and quantify heart disease in many different health care environments. Cardiac ultrasound imaging continues to evolve rapidly and further miniaturisation of digital technologies has recently led to the construction of marvels of modern electronic bioengineering: real time, three dimensional echocardiography and, at the other end of the spectrum, small hand carried imaging devices.

The basic physical examination as we practice it today was introduced by Pharaonic doctors and included history taking, inspection, palpation, and direct auscultation. In later times, doctors did not examine their patients. Renewed interest in the physical examination was stimulated by the pioneering work of GB Morgagni (1682-1771) who showed for the first time the pathologic changes induced in the organs by disease and how these cause signs and symptoms. Clinicians wanted to diagnose hidden pathology by detecting these signs at the bedside. It was the beginning of the "golden era" of physical diagnosis, with the great contributions of Auenbrugger, Corvisart, and Laennec. RTH Laennec (17811826) revolutionised the physical examination by the introduction of the stethoscope, the first technological aid in clinical medicine. In fact, stethoscope is a misnomer ( skopein = see) and stethophone (phone $=$ sound) would have been more appropriate.

\section{MILESTONES IN DIAGNOSTIC CAPABILITIES}

The stethoscope improved the quality of cardiac sounds and their perception by augmenting the sense of hearing. However, this technology and, almost a century later, $x$ ray imaging and electrocardiography were not directly accepted by clinicians as progress. Nevertheless, history shows that these technologies marked significant milestones in our diagnostic capabilities. W Rollins proposed in 1904 the "Seehear" device which extended the physical senses during the physical examination by simultaneously seeing the heart with a portable fluoroscope and hearing it with a stethoscope. This instrument was not implemented as a diagnostic aid, however, but the concept of seeing the invisible pathology during the physical examination was suggested.

It appears that we are still practising examination techniques that are 200 years old or more and that the younger generation of cardiologists increasingly relies on echo/Doppler as their primary method to increase their diagnostic abilities. Consequently, their examination skills are rapidly decreasing when compared to the standards of the "golden era" and to those of our master teachers, particularly in auscultation. ${ }^{1-4}$ There are several reasons for this decline in examination skills. It is generally recognised that the clinical diagnosis of many common cardiac conditions such as pericardial effusion, early ventricular dysfunction, early cardiomyopathy, silent valvar disease, and mass lesions is a challenge to the most experienced clinician or even impossible, while they are rapidly diagnosed by echo/ Doppler examination. ${ }^{5-7}$

It is also realised that an early aetiologic diagnosis often determines the most appropriate management plan and cardiologists increasingly appreciate the potential of echo/Doppler examination for establishing the cause of conditions. Unsuspected and not clinically apparent abnormalities are regularly detected by a simple echo/Doppler examination and may have an important bearing on therapeutic decisions and outcome. ${ }^{8}$ We all recognise that the standard physical examination has shortcomings in view of the contemporary paradigm shift to "early" and "pre-symptomatic" detection of disease. It has also been shown that the frequency of cardiovascular misdiagnosis in unselected patients who died in the hospital has halved over the past 20 years and has paralleled the increasing use of echo/Doppler examination.' In addition to these realities, the circumstances (noisy environment) in which we examine our patients, the many different clinical scenarios and pathologies, and more particularly the time constraints to perform a thorough physical examination have also changed considerably in recent years. All of these factors make us understand the changes in 
attitude of the younger generation of cardiologists. Is it not logical that they want to obtain as much objective information as possible when they first see the patients? Small hand carried ultrasound imaging devices offer the potential to achieve this desire. These devices can be used anywhere just like a conventional stethoscope. They should not replace the physical examination but significantly increase its yield and accuracy at first contact with the patient by extending our physical sense of seeing and recognising (major) cardiac abnormalities. ${ }^{6}{ }^{10-13}$

\section{WHO WILL USE THE HAND HELD DEVICES?}

However, the increasing availability of these small devices raises several important questions. How and by whom will these devices be used? Will they become an adjunct to the physical examination and will they ultimately be used by all clinicians including general practitioners? Or will they be further developed with more functions and merely used by experts as a substitute for standard echocardiography? Should there be specific training requirements?

It this issue, Quiles and colleagues ${ }^{14}$ report on their evaluation of the spectral Doppler modalities incorporated in one of the recent hand carried ultrasound imaging devices. Their study showed good agreement of the Doppler measurements between those obtained with the hand carried device and those with standard equipment. However, important limitations for the assessment of pulmonary artery pressure were noted. The investigators concluded that a complete assessment of valvar heart disease and diastolic left ventricular dysfunction is possible. Clearly, the Doppler functions incorporated in the small devices must be evaluated since they are now available and will be used in practice. However, I do believe that these expert investigators have come to a dangerous conclusion, not only because assessment of valvar heart disease requires estimation of pulmonary artery pressure and assessment of diastolic function involves more than the parameters tested in their study. More important is that their conclusion may stimulate the use of these devices as a substitute for standard echocardiography. This could be done by experts in certain clinical scenarios but it may also tempt cardiologists with few skills and limited experience to purchase these relatively low cost devices and perform examinations. The real danger is that these cardiologists may overstep the boundaries of good practice by improper use and misdiagnosis. This may damage both the acceptance and implementation of these devices for their most powerful application: augmenting the yield of the physical examination at the point-ofcare.

Clearly, it remains to be defined by whom and in what clinical setting the use of these devices will be most appropriate. I believe that these devices should not be further developed as smaller full featured systems. This will make them more complex, will require a higher level of training, and they may then generate more problems than they solve. There are already full featured portable systems available which can be used by experts but they are more costly. The small devices should be developed towards being smaller (pocket size), simpler to use, and cheaper, with good structure imaging and sensitive colour Doppler flow capabilities to allow the detection of major cardiac abnormalities (left ventricular dysfunction, left ventricular hypertrophy, valvar disease, effusion, masses) and for specific screening programmes. ${ }^{15-21}$ Whenever an abnormality is found, these patients must be referred for complete assessment. The yield of the physical examination with such a small device should be compared to the yield of the physical examination without. Undoubtedly, comparison of the cardiologist's knowledge with and without the information of the small imaging device will always be in favour of having this information.

\section{TRAINING REQUIREMENTS}

The application of these devices involves specific training requirements. If the system will be used as part of the physical examination, not every clinician should be trained in every aspect of echo/Doppler assessment, but the level of training could be defined to identify the major cardiac conditions and emergency problems. Limited training can also fulfil appropriate expectations for examination for a focused purpose-for example, screening. The size of these devices should not mean, however, that less training is required. Ideally, educational training should be incorporated in the medical student curriculum, just like stethoscopy is now; however, the cost of these devices is (still) a major limitation..$^{22}{ }^{23}$ Maintaining an acceptable level of competence may be a problem, although this is also true for the use of the standard stethoscope. The level of competence required may differ depending on the application and clinical scenario under consideration-for example, rapid screening and identifying acute problems in the critical care environment is different from answering referral questions in the outpatient clinic. The American Society of Echocardiography has recommended level 1 training for fellows and practising cardiologists. ${ }^{24}$ This may be sufficient for handling the transducer, operating the device, and recognising the major structural and flow abnormalities, but is insufficient for a complete assessment of valvar heart disease and diastolic dysfunction.

The satisfaction and the value of the physical examination so often neglected in our technological age may be rediscovered by the younger generation of cardiologists by the insights gained from using ultrasound stethoscopy and lead to a renaissance of the physical examination in the third millennium.

\section{REFERENCES}

1 Roldan CA, Shively BK, Crawford MH. Value of the cardiovascular physical examination for detecting valvular heart disease in asymptomatic subjects. Am J Cardiol 1996;77:1327-31.

2 Mangione S, Nieman LZ. Cardiac auscultatory skills of internal medicine and family practice trainees. A comparison of diagnostic proficiency. JAMA 1997;278:717-22.

3 Lok CE, Morgan CD, Ranganathan N. The accuracy and interobserver agreement in detecting the 'gallop sounds' by cardiac auscultation. Chest 1998;114:1283-8.

4 Attenhofer Jost $\mathrm{CH}$, Turina J, Mayer K, et al. Echocardiography in the evaluation of systolic murmurs of unkown cause. Am J Med 2000; 108:614-20.

5 Popp RL. The physical examination of the future: echocardiography as part of the assessment. ACC Current Rev 1998;7:79-81.

6 Vourvouri EC, Poldermans D, DeSutter J, et al. Experience with an ultrasound stethoscope. J Am Soc Echocardiogr 2002;15:80-5.

7 Roelandt JRTC. A personal ultrasound imager (ultrasound stethoscope). A revolution in the physical cardiac diagnosis! Eur Heart J 2002;23:523-27.

8 Bossone E, DiGiovine B, Watts S, et al. Range and prevalence of cardiac abnormalities in patients hospitalised in a medical ICU. Chest 2002; 1 22:1370-6

9 Sonderegger-Iseli K, Burger S, Muntwyler J, et al. Diagnostic errors in three medical eras: a necropsy study. Lancet 2000;355:2027-31.

10 Rugolotto M, Hu BS, Liang DH, et al. Rapid assessment of cardiac anatomy and function with a new hand-carried ultrasound device (OptiGo): a comparison with standard echocardiography. Eur J Echocardiogr 2001;2:262-9.

11 Rugolotto $M$, Chang $C P, H u B$, et al. Clinical use of cardiac ultrasound performed with a hand-carried device in patients admitted to acute cardiac care. Am J Cardiol 2002;90:1040-2.

12 Spencer KT, Anderson AS, Bhargava A, et al. Physician-performed point-of-care echocardiography using a laptop platform compared with physical examination in the cardiovascular patient. J Am Coll Cardiol 2001;37:2013-8.

13 Bruce CJ, Montgomery SC, Bailey KR, et al. Utility of hand-carried ultrasound devices used by cardiologists with and without significant echocardiographic experience in the cardiology inpatient and outpatient settings. Am J Cardiol 2002:90:1273-5.

14 Quiles J, Garcia-Fernandez MA, Almeida PB, et al. Portable spectral Doppler echocardiographic device: overcoming limitations. Heart 2003;89:1014-8

15 Kimura BJ, Amundson SA, Willis CL, et al. Usefulness of a hand-held ultrasound device for bedside examination of left ventricular function. Am J Cardiol 2002;90:1038-9. 
16 Vourvouri EC, Poldermans D, Schinkel AFL, et al. Abdominal aortic aneurysm screening using a hand-held ultrasound device. A pilot study. Eur J Vasc Endovasc Surg 2001;22:352-4.

17 Goodkin GM, Spevack DM, Tunick PA, et al. How useful is hand-carried bedside echocardiography in critically ill patients? J Am Coll Cardiol 2001;37:2019-22

18 Galasko GIW Lahiri A, Senior R. Portable echocardiography: an innovative tool in screening for cardiac abnormalities in the community. Eur J Echocardiogr 2003:4:119-27.

19 Kimura BJ, Scott R, Willis CL, et al. Accuracy and cost-effectiveness of single-view echocardiographic screening for suspected mitral valve prolapse. Am J Med 2000;108:331-3.

20 Sheps SG, Frohlich ED. Limited echocardiography for hypertensive left ventricular hypertrophy. Hypertension 1997;29:560-3.
21 Bruce CJ, Spittell PC, Montgomery SC, et al. Personal ultrasound imager: abdominal aortic aneurysm screening. J Am Soc Echocardiogr 2000;13:674-9

22 Alexander JH, Peterson ED, Chen AY, et al. Training and accuracy of non-cardiologists in simple use of point-care-echo: a preliminary report from the Duke limited echo assessment project (LEAP). Thoraxcentre J 2001:13:105-10.

23 Wittich CM, Montgomery SC, Neben MA, et al. Teaching cardiovascular anatomy to medical students by using hand-held ultrasound device. JAMA 2002;288: 1062-3.

24 Seward JB, Douglas PS, Erbel R, et al. Hand-carried cardiac ultrasound $(\mathrm{HCU})$ device: recommendations regarding new technology. A report from the echocardiography task force on new technology of the nomenclature and standards committee of the American Society of Echocardiography. J Am Soc Echocardiogr 2002;15:369-73.

\section{IMAGES IN CARDIOLOGY}

\section{Percutaneous balloon fenestration of the intimal flap for management of limb threatening ischaemia in acute aortic dissection}

A 61 year old man with a history of systemic hypertension was admitted with severe chest and back pain of acute onset. Contrast enhanced computed tomography (CT) revealed acute Stanford type B aortic dissection extending to the iliac arteries. The patient was initially stabilised by intravenous antihypertensive medication, but soon developed critical right sided limb ischaemia. For restoration of perfusion, percutaneous balloon fenestration was considered. Angiography showed complete occlusion of the right common iliac artery (CIA) at the level of the aortic bifurcation (arrow; upper panel, left; TL, true lumen). The intimal flap was punctured from the true lumen using a Brockenborough needle and a guide wire was passed into the false lumen. A balloon with a diameter of $14 \mathrm{~mm}$ was used to enlarge the puncture site within the intimal flap (upper panel, right). Although aortography demonstrated communication between true and false lumen, flow into the right common iliac artery remained impaired. Additional stent placement (arrows) into the right common iliac artery was required to re-establish limb perfusion (lower panel, left; FL, false lumen). Following the procedure, the patient developed reperfusion syndrome with a maximal myoglobin of $155742 \mu \mathrm{g} / \mathrm{l} \quad$ requiring transient haemodialysis.

After recovery of the patient, endovascular stent-graft placement was performed to seal the proximal entry tear in the thoracic aorta. The patient was finally discharged six months after the
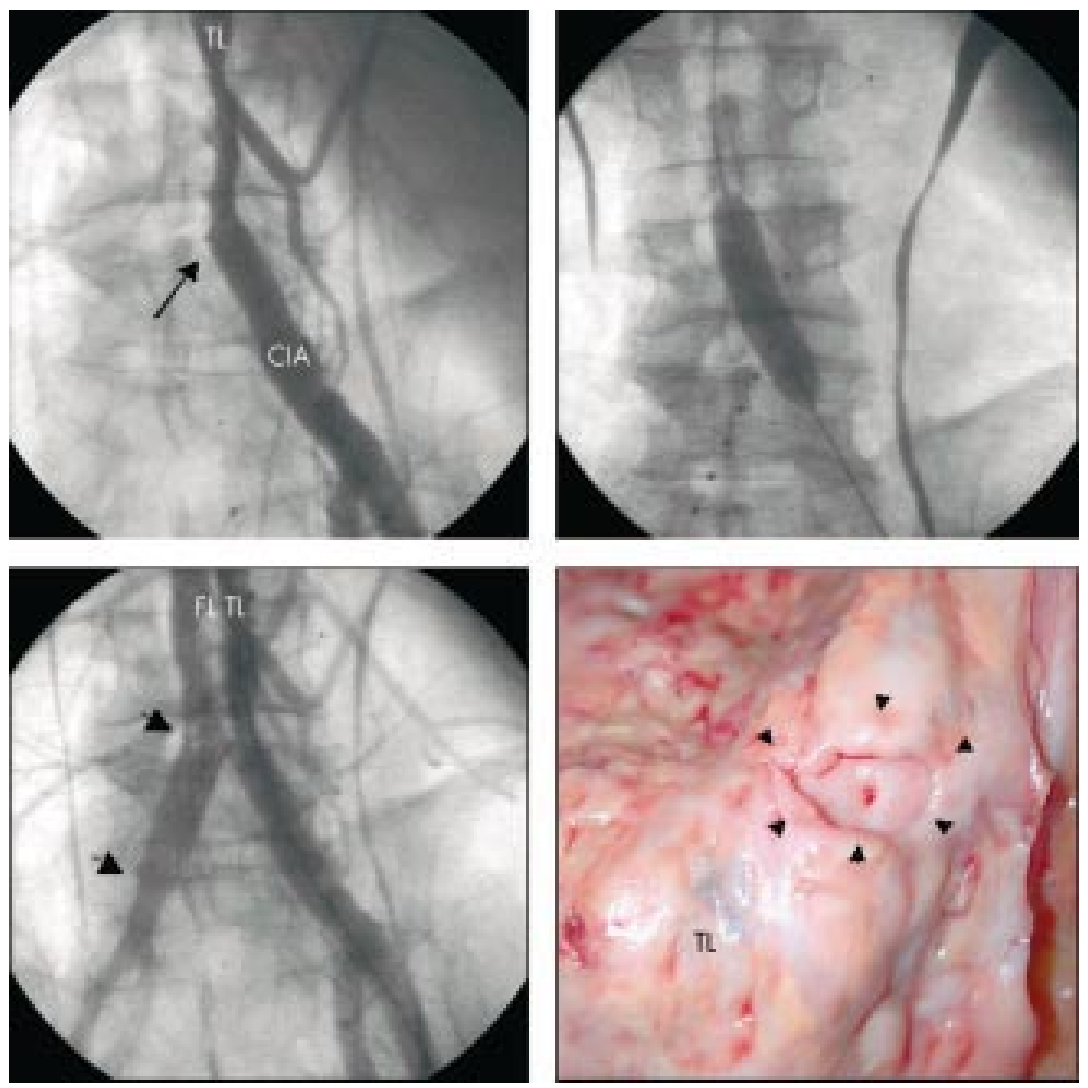

initial event. However, he died from sep-

H Eggebrecht

D Baumgart

O Dirsch

charge. At necropsy, the large tear in the intimal flap created by balloon fenestration could be seen (arrows; lower panel, $R$ Erbel right). 